\title{
Percepção sobre saúde bucal e a importância do acompanhamento odontológico durante o período gestacional e puerperal
}

\author{
Perception about oral health and the importance of dental care during pregnancy and \\ puerperal period
}
Percepción sobre la salud bucal y la importancia del cuidado dental durante el embarazo y el período puerperal

Ana Larissa Fernandes de Holanda Soares ${ }^{1 *}$, Chauí de Lima Cabral ${ }^{1}$, Sanny da Silva Fonseca Beserra $^{1}$, Renata Taise da Costa Feliciano Santiago ${ }^{1}$, Fernando Jales Borges ${ }^{1}$, Isaías Lopes de Medeiros $^{1}$, Layza Tércia Marcelino Leite ${ }^{1}$, Juliana Fernandes da Silva ${ }^{1}$, Hiago Bruno Pereira de

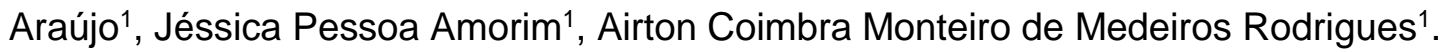

\section{RESUMO}

Objetivo: Discutir sobre o acompanhamento odontológico e a importância da saúde bucal no período gestacional e puerperal. Métodos: Estudo transversal, descritivo, exploratório e quantitativo realizado em uma maternidade na capital do Rio Grande do Norte. Participaram desta pesquisa 72 mulheres - gestantes e puérperas - que estavam internadas na maternidade durante o período da coleta de dados (abril de 2018/maio de 2018). Resultados: Os resultados apontaram que $75 \%$ das entrevistadas não receberam informações de saúde bucal na gravidez e, das que receberam, a principal fonte de informações foi o profissional dentista; $61 \%$ das gestantes e puérperas informaram acreditar que a gravidez em si pode ocasionar algum problema na cavidade oral. Dentre os problemas bucais mais apontados estão o sangramento gengival, a sensibilidade dentária e dente enfraquecido. Conclusão: $O$ pensamento de que grávida não pode ter assistência odontológica foi o principal mito identificado. Assim, acentuamos a importância de uma maior inserção da equipe de saúde bucal nos trabalhos multi e interprofissionais, com atuação de promoção e proteção da saúde e prevenção de doenças para desmistificar o acompanhamento odontológico na gestação e melhorar a percepção deste público para com sua saúde bucal.

Palavras-chave: Gravidez, Saúde bucal, Dentista.

\begin{abstract}
Objective: To discuss dental care and the importance of oral health during pregnancy and puerperal period. Methods: Cross-sectional, descriptive, exploratory and quantitative study carried out in a maternity hospital in the capital of Rio Grande do Norte. 72 women participated in this research - pregnant women and puerperal women - who were hospitalized in the maternity hospital during the period of data collection (April 2018 / May 2018). Results: The results showed that $75 \%$ of the interviewees did not receive oral health information during pregnancy and, of those who did, the main source of information was the dentist; $61 \%$ of pregnant women and puerperal women reported believing that pregnancy itself can cause some problem in the oral cavity. Among the most pointed oral problems are gingival bleeding, tooth sensitivity and weakened teeth. Conclusion: The thought that pregnant women cannot have dental care was the main myth identified. Thus, we emphasize the importance of a greater insertion of the oral health team in multi and interprofessional work, with activities to promote and protect health and prevent diseases in order to demystify dental care during pregnancy and improve this public's perception of their oral health.
\end{abstract}

Keywords: Pregnancy, Oral health, Dentist.

1 Universidade Potiguar (UnP) Natal - RN. *E-mail: chaui786@gmail.com 


\section{RESUMEN}

Objetivo: Discutir el cuidado dental y la importancia de la salud bucal durante el embarazo. Métodos: Estudio transversal, descriptivo, exploratorio y cuantitativo realizado en una maternidad de la capital de Rio Grande do Norte. 72 mujeres participaron en esta investigación, mujeres embarazadas y mujeres puerperales, que fueron hospitalizadas en el hospital de maternidad durante el período de recopilación de datos (abril de 2018 / mayo de 2018). Resultados: Los resultados mostraron que el $75 \%$ de las entrevistadas no recibieron información de salud oral durante el embarazo y, de quienes sí lo hicieron, la principal fuente de información fue el dentista; El $61 \%$ de las mujeres embarazadas y las mujeres puerperales informaron creer que el embarazo en sí mismo puede causar algún problema en la cavidad oral. Entre los problemas orales más agudos están el sangrado gingival, la sensibilidad dental y los dientes debilitados. Conclusión: La idea de que las mujeres embarazadas no pueden recibir atención dental fue el principal mito identificado. Por lo tanto, enfatizamos la importancia de una mayor inserción del equipo de salud bucal en el trabajo multi e interprofesional, con actividades para promover y proteger la salud y prevenir enfermedades a fin de desmitificar la atención dental durante el embarazo y mejorar la percepción de este público sobre su salud bucal.

Palabras clave: Embarazo, Salud bucal, Dentista.

\section{INTRODUÇÃO}

A gravidez é um período delicado na vida de uma mulher e o nascimento do filho é uma experiência única, portanto merecem ser tratados de forma singular e especial por profissionais qualificados e pela equipe multiprofissional (VIEIRA SM, et al, 2011). As gestantes são consideradas pacientes especiais por ser um grupo de risco para doenças bucais e também pelo fato de apresentarem alterações físicas, biológicas e hormonais que acabam por criar condições adversas no meio bucal (ROSSEL FL, 1998). Ainda nos dias hodiernos, o tratamento odontológico à gestante é rodeado de crenças, mitos e muitas vezes visto também como algo negativo, fazendo com que a busca pelos cuidados em saúde por parte da mulher grávida dificilmente aconteça.

A falta de conhecimento e de informação, o medo do dentista, a crença de que a dor estar associada à gravidez, a incerteza de um tratamento durante esta época, dentre outros, são relatados em diversos estudos como fatores contribuintes para a ausência de buscas de ajuda odontológica. Além disso, existem profissionais dentistas que apresentam despreparo na hora de dar assistência, chegando até mesmo a adiar tratamentos para depois do parto, podendo assim causar deterioração da saúde bucal, bem como injuriar a saúde geral destas pacientes (ALVES RT, et al., 2010; CODATO LAB, et al., 2011; NACIMENTO EP, et al., 2012).

A falta de integração multi e interprofissional e a falta de priorização da saúde bucal também são consideradas entraves no acompanhamento odontológico das gestantes. Os cuidados com a saúde bucal ainda são vistos como uma atividade à parte e infelizmente o profissional dentista muitas vezes não é tido como parte de uma equipe multiprofissional (LEAL NP e JANOTTI CB, 2009).

Codato LAB, et al. (2011) explica que, por ser um período de muitas mudanças, a gravidez torna-se uma etapa favorável para a promoção de saúde, pela possibilidade de estabelecimento, incorporação e mudanças de hábitos, já que a gestante tende a ser estimulada a buscar informações nessa época. Assim é possível que se obtenham melhorias no autocuidado da gestante em relação à saúde bucal e consequente diminuição do aparecimento de problemas bucais como cárie e doença periodontal.

A atuação de maneira interprofissional em saúde é importantíssima, pois ela é capaz de influenciar os determinantes do processo saúde-doença bucal. Assim, como a mulher durante a gravidez se apresenta psicologicamente mais receptiva a novos conhecimentos, poderá haver melhores condições de vida tanto para ela como para o bebê, tendo em vista que os conhecimentos sobre promoção da saúde que são repassados influenciam de modo positivo na formação de hábitos saudáveis (ARAÚJO SM, et al., 2009). 
Partindo dessa premissa, o objetivo do presente trabalho foi discutir sobre o acompanhamento odontológico e a importância da saúde bucal na gravidez, além de identificar os principais mitos existentes segundo as gestantes e puérperas que estavam internadas em uma maternidade na capital do Rio Grande do Norte durante o período de coleta de dados.

\section{MÉTODOS}

A pesquisa foi caracterizada como um estudo transversal, descritivo, exploratório e quantitativo realizado em uma maternidade na capital do Rio Grande do Norte. A amostragem foi por conveniência e, portanto, não probabilística. Participaram desta pesquisa 72 mulheres - gestantes e puérperas - que estavam internadas na maternidade durante o período da coleta de dados (abril de 2018/maio de 2018). Foram incluídas todas as gestantes e puérperas que concordaram em assinar o Termo de Consentimento Livre e Esclarecido, autorizando a sua participação no estudo. Não houve critérios de exclusão.

A coleta de dados aconteceu por meio da aplicação de um questionário semiestruturado, individualmente e de forma oral, com 14 perguntas, algumas delas abertas. O questionário foi composto por perguntas sobre dados gerais das participantes, questões socieconômicas, saúde bucal, pré-natal e acompanhamento odontológico durante a gravidez. O instrumento de coleta utilizado foi validado a partir de um estudo piloto na mesma maternidade. Os dados foram analisados de forma descritiva, a partir de tabelas e gráficos. $O$ programa utilizado foi o Excel® 2007.

O presente trabalho foi aprovado no Comitê de Ética e Pesquisa da Universidade Potiguar (UnP) (parecer 2.475.470) e obedeceu às normas éticas exigidas pela Resolução no 466/2012 do Conselho Nacional de Saúde. Após todo esclarecimento acerca da pesquisa, as gestantes e puérperas que aceitaram participar da mesma assinaram o Termo de Consentimento Livre e Esclarecido.

Toda pesquisa com seres humanos envolve risco em tipos e gradações variados. O presente estudo vislumbrou como possível risco emocional o constrangimento dos participantes. Dessa forma, para minimizar esse risco, os questionários foram aplicados individualmente e em um local reservado de cada sala. $O$ benefício foi o conhecimento sobre os mitos existentes na sociedade, para esclarecimento a respeito, bem como o conhecimento da importância do trabalho multi e interprofissional acerca do ciclo gravídico das mulheres.

\section{RESULTADOS E DISCUSSÃO}

A faixa etária mais preponderante neste estudo $(61,1 \%)$ foi entre 19 e 29 anos, o que está de total acordo com as pesquisas de Rosell FL, et al. (2013) onde a maioria de suas entrevistadas apresentaram a mesma faixa etária - que é o período considerado pela literatura como fértil para a mulher engravidar. Quanto a escolaridade, a maioria das participantes (34\%) possuía ensino médio completo ou incompleto (Gráfico 1), diferente do estudo de Mesquita BS, et al. (2013), onde a maioria $(51,11 \%)$ de suas entrevistadas apresentava ensino fundamental completo/incompleto.

Gráfico 1 - Grau de escolaridade das participantes da pesquisa.

Grau de Escolaridade das participantes da pesquisa

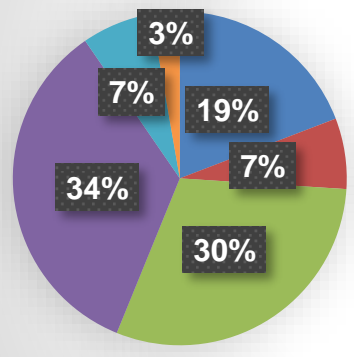

$$
\begin{aligned}
& \text { Ensino fundamental incompleto } \\
& \text { Ensino fundamental completo } \\
& \text { Ensino médio incompleto } \\
& \text { Ensino médio completo } \\
& \square \text { Ensino superior incompleto } \\
& \text { Ensino superior completo }
\end{aligned}
$$

Fonte: Soares ALFH, et al., 2018. 
Tanto a idade quanto o nível educacional possuem vínculo com a saúde, já que podem influenciar significativamente na cooperação dos pacientes. Esse vínculo entre saúde e condições socioambientais muitas vezes serve de base para trabalhos multi e interprofissionais nas comunidades, onde abordam prevenção, educação, conhecimento e cultura, coisas que devem ser impregnadas durante a formação dos indivíduos desde cedo, cabendo ao profissional de saúde ser um facilitador desse processo educativo (NOGUEIRA LT, et al., 2012).

No que concerne à quantidade de pessoas que moravam na mesma residência das entrevistadas, as respostas mais frequentes foram "04" e "05" pessoas. E como principal ocupação, $36,1 \%$ englobava os afazeres do lar, resultado que corrobora com o estudo de Cabral MCB, et al. (2013), onde a maioria de suas participantes (46,3\%) tinham como ocupação principal os afazeres domésticos.

Das entrevistadas, apenas $31(56 \%)$ estavam em sua primeira gravidez, algo semelhante com o estudo de Bastiani C, et al. (2010), no qual enfatizaram que ser mãe de primeira viagem é uma característica importante, pois mulheres primigestas são psicologicamente mais receptivas a informações referente aos cuidados relacionados com sua saúde, sendo assim mais disposta a mudar padrões que provavelmente terão influência no desenvolvimento da sua saúde e do seu bebê.

Quanto ao recebimento de informações em saúde bucal durante a primeira ou em alguma das gestações, $54(75 \%)$ respondeu que não recebeu nenhum tipo de informação, enquanto que 18 (25\%) recebeu. Das que receberam, o profissional de saúde mais frequentemente mencionado como responsável por passar tais informações foi o próprio dentista (57\%), seguido do médico (24\%), do enfermeiro (9\%) e ainda de outros profissionais (10\%) (Gráfico 2). Quanto às orientações, 15 delas (63\%) receberam sobre higiene bucal, 4 (17\%) sobre saúde bucal do bebê, $2(8 \%)$ sobre cárie, apenas $1(4 \%)$ sobre doença periodontal e $4(8 \%)$ sobre outros assuntos não categorizados pela pesquisa.

Gráfico 2 - Respostas referentes ao profisional responsável por orientar sobre saúde bucal durante a gravidez.

Respostas referentes ao profisional responsável por orientar sobre saúde bucal durante a gravidez

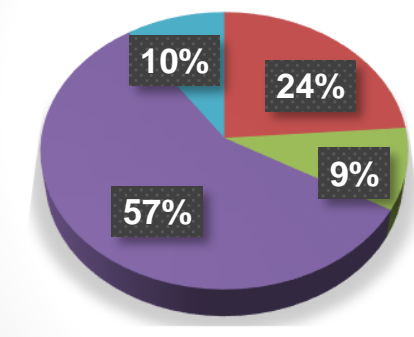

$$
\begin{aligned}
& \text { - Profissional } \\
& \text { - Médico } \\
& \text { - Enfermeiro } \\
& \text { - Dentista } \\
& \text { - Outro }
\end{aligned}
$$

Fonte: Soares ALFH, et al., 2018.

Batistella FID, et al. (2006), observaram em sua pesquisa que a maioria das suas entrevistadas $(64,4 \%)$ também não recebeu informação sobre saúde bucal durante a gravidez. Segundo as que receberam, o profissional dentista (34,9\%) também foi o mais aludido como fonte de informação.

Quanto ao pré-natal, $42(59 \%)$ disse ter começado a usufruí-lo quando estava acerca do primeiro ou segundo mês de gestação, 24 (33\%) a partir do terceiro ou quarto mês e $6(8 \%)$ por volta do quinto ou sexto mês. Porém, ao serem questionadas sobre a presença do dentista no pré-natal, a resposta foi unânime todas as entrevistadas responderam como importante e as justificativas mais apontadas foram que 0 profissional dentista seria capaz de passar informações sobre saúde bucal da gestante e do bebê, onde elas estariam, consequentemente, mais informadas $(41,6 \%)$; trataria os problemas dentários durante a gravidez $(54,1 \%)$; e $30,5 \%$ respondeu que preveniria doenças ou agravos na cavidade oral da gestante e do bebê (Tabela 1). Apesar da unanimidade, somente $13(18 \%)$ das entrevistadas disse ter sido acompanhada pelo dentista durante o pré-natal. 
Tabela 1 - Valores absolutos dos tipos de justificativa sobre a importância do acompanhamento odontológico durante a gestação.

\begin{tabular}{ccc}
\hline \multicolumn{1}{c}{ Justificativa } & № & $\%$ \\
\hline $\begin{array}{c}\text { Ficaria mais informada sobre a sua saúde bucal e a do } \\
\text { Bebê }\end{array}$ & $30 / 72$ & $41,6 \%$ \\
\hline $\begin{array}{c}\text { Trataria os problemas dentários na gravidez } \\
\text { Preveniria doenças ou agravos na cavidade oral da } \\
\text { Gestante e do bebê }\end{array}$ & $39 / 72$ & $54,1 \%$ \\
\hline
\end{tabular}

Fonte: Soares ALFH, et al., 2018.

Fernandes RA, Narchi NZ (2008) e Goepel E, et al. (1991) explicaram que as gestantes desconhecem fatores biomédicos cruciais a sua saúde bucal e possuem percepções arraigadas na cultura popular à respeito de doenças bucais e gravidez, sendo um dos motivos para não colocarem o pré-natal odontológico como prioridade, contribuindo assim para a precariedade das condições bucais, tanto da mãe quanto do bebê.

Santos CJGB (2010) reforçou em seu trabalho a importância de se realizar o pré-natal odontológico, onde o acompanhamento pelo dentista e a participação dos outros profissionais de saúde bucal durante a gestação podem fornecer as ferramentas necessárias para que as gestantes possam manter uma rotina que lhes propicie qualidade de vida, pois os profissionais de saúde bucal ao se inserirem no trabalho com gestantes podem conscientizá-las sobre o quanto é importante o desenvolvimento de hábitos saudáveis para si e para as futuras gerações e até desmistificar crenças que foram espalhadas na sociedade de maneira cultural sobre esse contexto. Desta forma, torna-se fundamental o incentivo ao desenvolvimento de hábitos de higiene bucal saudáveis, e de uma alimentação balanceada a estas pacientes.

Quanto à época ideal para atendimento odontológico, 22\% delas acreditavam que somente após o $3^{\circ}$ mês de gravidez, $4 \%$ respondeu que em nenhuma época e $4 \%$ em outra época, enquanto $60 \%$ achava que a gestante poderia ser atendida durante toda a gestação, sendo a resposta mais apontada entre as entrevistadas.

O que está de acordo com o estudo de Catarin RFZ, et al. (2008) que explicaram não haver nenhuma contraindicação referente ao atendimento odontológico durante toda a gestação, principalmente nos casos de urgência que visam à remoção da dor e/ou focos de infecção, uma vez em que a septicemia é teratogênica e pode causar aborto espontâneo. Contudo, as autoras deixaram claro que o segundo trimestre é tido como o mais confortável para atendimentos odontológicos.

O Ministério da Saúde (2008) publicou que seria contraindicada a realização de tratamentos odontológicos, tanto no primeiro quanto no terceiro trimestre da gestação. Argumentou que o primeiro é considerado como o menos adequado por ser um período em que há as principais transformações embriológicas; no terceiro, existe uma possibilidade maior da gestante sofrer síncope (devido compressão da veia cava inferior), apresentar hipertensão e anemia e por isso se deve evitar fazer procedimentos nesse período. Sobre as urgências, disse que as grávidas deveriam ser atendidas mediante os cuidados indicados em cada período gestacional. Já quanto ao segundo trimestre, o Ministério da Saúde (2008) também defendeu ser o mais adequado para a realização de intervenções clínicas e procedimentos odontológicos essenciais;

Quando questionadas sobre a existência de medo ou não na realização de procedimentos odontológicos durante a gravidez, 10,6\% relataram não ter medo de nenhum procedimento e $44 \%$ disse não ter realizado nenhum procedimento. Das que relataram ter medo, a anestesia local odontológica $(16,8 \%)$ e a extração $(13 \%)$ foram os mais apontados, seguidos de canal $(6,5 \%)$, radiografia $(6,5 \%)$ e outros $(2,6 \%)$. O que coincide com o estudo de Scavuzzi AIF, et al. (2008) em que também a anestesia e a extração foram os mais apontados como procedimentos que mais causavam medo nas participantes por acreditarem que estes eram danosos ao bebê. 
Trevisan CL e Pinto AAM (2013) reforçaram que as representações sobre o tratamento odontológico na sociedade envolvem imagens negativas que geram tais medos e até ansiedade onde, na gravidez, podem ser potencializados. Eles ainda explicaram que, por parte das gestantes, poderia existir certa resistência ao acompanhamento odontológico que está fundada em crenças muito difundidas em nosso meio de que esses procedimentos influenciem no curso da gestação e provoquem danos à mãe e/ou ao bebê.

A respeito de atendimento odontológico recusado, apenas $8(11 \%)$ disse que sim, onde relataram que as justificativas dadas pelos dentistas foram: $(40 \%)$ que não se sentia preparado, $(20 \%)$ não tinha o equipamento necessário e (40\%) outra justificativa: neste último obtivemos respostas como: "não era bom realizar o atendimento por causa do bebê"; "... não me atendeu por causa que eu estava no $8^{\circ}$ mês de gravidez..."; "...porque eu tava no início da gestação..."; "...não tinha a anestesia apropriada..."; "...porque eu era gestante e que só poderia após o $7^{\circ}$ mês da gravidez...".

Muitas destas justificativas são chocantes, tendo em vista que vão de contramão à Portaria $n^{\circ} 2.436$ de 21 de setembro de 2017, a qual diz que fica proibida qualquer tipo de exclusão baseada em idade, gênero, cor, crença, nacionalidade, etnia, orientação sexual, identidade de gênero, estado de saúde, condição socioeconômica, escolaridade ou limitação física, intelectual, funcional, entre outras (BRASIL, 2017).

Tais justificativas também vão contra a Portaria $n^{\circ} 1.459$, de 24 de junho de 2011 que institui a Rede Cegonha, onde em seu artigo $1^{\circ}$ diz que, instituída no âmbito do Sistema Único de Saúde, tal Rede promove um gama de cuidados que visam assegurar à mulher o direito ao planejamento reprodutivo e à atenção humanizada à gravidez, ao parto e ao puerpério, bem como o direito ao nascimento seguro e ao crescimento e ao desenvolvimento saudáveis da criança (BRASIL, 2011)

O despreparo do dentista identificado neste trabalho provavelmente foi uma das principais razões para essa recusa de atendimento odontológico à gestante. O que se assemelha com os achados de Codato LAB, et al. (2008) e Maeda FHL, et al. (2001), onde destacaram que ainda acontece de muitos profissionais protelarem 0 atendimento às mulheres grávidas pelo receio que sentem de serem responsabilizados por possíveis fatalidades que possam futuramente vir a ocorrer com o bebê, além de muitas vezes se sentirem inseguros diante de mitos existentes acerca deste contexto, isso possivelmente pela falta de prática/domínio acerca deste contexto desde a época de graduação.

Cerca de $61 \%$ das gestantes e puérperas informaram acreditar que a gravidez em si pode ocasionar algum problema na cavidade oral. Dentre os problemas bucais mais apontados estão o sangramento gengival, a sensibilidade dentária e dente enfraquecido (Tabela 2). Também foram mencionados outros problemas como cárie, dor e perda dentária, semelhante a pesquisa de Bastiani C, et al. (2010), onde 35\% de suas entrevistadas afirmaram que os dentes ficam mais fracos na gestação.

Tabela 2 - Valores absolutos e percentuais dos tipos de problemas bucais que a gravidez pode desencadear, na percepção das participantes.

\begin{tabular}{ccc}
\hline Justificativa & № & $\%$ \\
\hline Cárie & 09 & $12,5 \%$ \\
\hline Sangramento gengival & 19 & $26,6 \%$ \\
\hline Enfraquecimento do elemento dentário & 14 & $19,4 \%$ \\
\hline Perda de dente & 01 & $1,4 \%$ \\
\hline Dor de dente & 23 & $31,9 \%$ \\
\hline Não resposta & 06 & $8,2 \%$ \\
\hline
\end{tabular}

Fonte: Soares ALFH, et al., 2018.

Nesse sentido, Martins LO, et al. (2013) disseram ser necessária uma maior dedicação por parte dos profissionais dentistas na transmissão de informações corretas às gestantes acerca desse contexto, tendo em vista que grande parcela da população acredita que a gravidez causa problemas bucais. 
É importante destacar que a gestação em si não é causadora de problemas bucais, mas as alterações fisiológicas da gravidez poderiam agravar problemas pré-existentes, sobretudo em mulheres carentes, porque, em geral, são as que menos procuram assistência odontológica preventiva (FERNANDES RA e NARCHI NZ, 2008).

64\% das participantes deste estudo relataram não ter ingerido açúcar durante a gravidez, ao passo em que $36 \%$ ingeriu. Finkler M, et al. (2004) realizaram um estudo qualitativo que aludiu as representações sociais das gestantes referente à saúde bucal do bebê, e também identificaram que uma parcela de suas entrevistadas disse ter feito consumo racional do açúcar no período gestacional, onde inclusive mencionaram até a relação cárie-alimentos cariogênicos e o cuidado com a transmissibilidade de microrganismos entre mãe e filho, aparentando ser afirmações conscientes.

Algumas das nossas entrevistadas, como visto anteriormente, relataram acreditar que a gravidez em si pudesse ocasionar cárie, entretanto não mencionaram nada referente à associação alimento cariogênicodoença cárie, o que nos leva a supor que as que assumiram ter feito ingestão exagerada de açúcar durante a gravidez não estavam preocupadas com as consequências que tal ato poderia trazer para elas e/ou bebê, ou que não tiveram nenhum tipo de informação em saúde sobre isso.

Quando questionadas se haviam sentido dor de dente ou algum incômodo dentário durante a gravidez, $42 \%$ disseram que sim. Dessas, mais de 50\% informou não ter buscado o dentista para identificar o problema, externando motivos como: era uma dor dava para suportar, automedicação e medo de ir ao dentista. Esses achados são parecidos com os da pesquisa de Rosa PC, et al. (2007), onde também constataram que muitas de suas participantes já tinham sentido dor de dente durante a gravidez, mas que também não se dispuseram a buscar o dentista para identificar a causa.

Silva ADM (2001) enfatizou que havia uma aceitação passiva e inquestionável por parte das gestantes, da "necessidade" de esperar o nascimento do bebê para "poder" iniciar ou retomar algum tratamento odontológico. Ela ainda explicou que nem mesmo a dor às vezes supera o medo e a resistência ao tratamento odontológico durante a gestação. Quanto aos resultados desta pesquisa a respeito de dor de dente durante a gravidez, essas são provavelmente as principais explicações.

$82 \%$ de nossas entrevistadas disseram não ter se automedicado, onde apresentaram como razões para tal: preferir tomar medicamento com autorização/consentimento do médico, não gostar de se automedicar, acreditar que a grávida não podia tomar nenhum medicamento e outros motivos. Enquanto isso, $18 \%$ informou ter ingerido algum tipo de medicamento na gravidez sem a prescrição de algum profissional da saúde, sendo os fármacos das classes analgésica $(91,4 \%)$ e antiespasmódica $(7,6 \%)$, paracetamol e buscopan respectivamente, os mais utilizados pelas participantes desta pesquisa. $\mathrm{O}$ que divergiu um pouco do estudo de Fonseca MRCC, et al. (2002) em que a maioria de suas entrevistadas $(52,8 \%)$ informaram ter feito o uso indiscriminado de fármacos da classe de antiácidos, seguidos dos medicamentos analgésicos $(34,3 \%)$.

É importante destacarmos que é muito perigoso o ato da automedicação, principalmente quando se é gestante, tendo em vista que diversos tipos de medicamentos podem causar injúrias a saúde da grávida e do bebê. Zanrosso CW, et al. (2010) explicam que a exposição materna a dipirona durante o período gestacional, por exemplo, pode ser considerada como um fator de risco para o desenvolvimento de doenças na infância, contraindicando assim seu uso neste período, pois esse medicamento apresenta Categoria D na classificação da Food and Drug Admnistration.

As participantes deste estudo ainda citaram ter feito automedicação de outros grupos de fármacos como o lbuprofeno, um anti-inflamatório não esteroidal (AINES) e também analgésico, que, segundo a pesquisa de Chai WL e Ngeow WC (1998), tem potencial de teratogenicidade e possivelmente tem associação com efeitos deletérios sobre a circulação fetal e neonatal. Suresh $L$ e Radfar $L$ (2004) ainda reforçaram que o uso dos AINES não é recomendável devido aos seus efeitos adversos relativamente comuns que também podem ocorrer na gestante, como complicações gastrointestinais.

Dentre as limitações da nossa pesquisa, pode-se destacar a falta da informação sobre possuir a caderneta da gestante, pois essa pergunta não foi incluída no questionário, a amostragem de conveniência, o que não permite a inferência estatística e se trata de um estudo transversal, impossibilitando fechar a causalidade. 


\section{CONCLUSÃO}

Ainda existem enraizados culturalmente na sociedade diversos mitos e crenças a respeito do acompanhamento odontológico na gravidez. Dessa forma, concluímos que o pensamento de que gravida ou a puérpera não pode ter assistência odontológica foi o principal mito identificado. Assim, acentuamos a importância de uma maior inserção não somente do dentista, mas de toda a equipe de saúde bucal nos trabalhos multi e interprofissionais, com atuação de promoção e proteção da saúde, bem como prevenção de doenças e agravos para assim desmistificar o acompanhamento odontológico na gestação e melhorar a percepção deste público para com sua saúde bucal.

\section{REFERÊNCIAS}

1. ALVES RT, et al. Perfil epidemiológico e atitudinal de saúde bucal de gestantes usuárias do serviço público de Juiz de Fora, MG. Pesq. Bras. Odontoped Clin. Integr., 2010; 10(3): 413-421.

2. ARAUJO SM, et al. Conhecimento e atitudes dos médicos ginecologistas/obstetras a respeito da saúde bucal da gestante. RFO. 2009; 4(3): 190-196.

3. BASTIANI C, et al. Conhecimento das gestantes sobre alterações bucais e tratamento odontológico durante a gravidez. Odontol. Clín. Cient. 2010; 9(2): 155-160.

4. BATISTELLA FID, et al. Conhecimento das Gestantes Sobre Saúde Bucal. Revista Gaúcha de Odontologia, P. Alegre. 2006; 54(1): 67-73.

5. BRASIL. Portaria no 2436, de 21 de setembro 2017. Aprova a Política Nacional de Atenção Básica, estabelecendo a revisão de diretrizes para a organização da Atenção Básica, no âmbito do Sistema Único de Saúde (SUS).

6. BRASIL. Ministério da Saúde. Caderno de Atenção Básica n 17. Brasília, DF, 2008. 65p.

7. BRASIL. Ministério da Saúde. Portaria n 1.459, de junho de 2011.

8. CABRAL MCB, et al. Percepção das gestantes do Programa de Saúde da Família em relação à saúde bucal no município de Ribeirópolis, Sergipe, Brasil. Revista Portuguesa de Saúde Pública. 2013; 31.

9. CATARIN RFZ, et al. Conhecimentos, práticas e acesso a atenção à saúde bucal durante a gravidez. Espaç. Saúde (Online). 2008; 10(1): 16:24.

10. CHAI WL e NGEOW WC. Dental care for pregnant patients: a reappraisal. Annals Dent. Univ. Malaya. 1998.

11. CODATO LAB, et al. Atenção odontológica à gestante: papel dos profissionais de saúde. Ciênc. saúde coletiva. 2011; 16.

12. CODATO LAB, et al. Percepções de gestantes sobre atenção odontológica durante a gravidez. Ciênc.saúde coletiva. 2008; 13.

13. FERNANDES RA e NACHI NZ. Saúde bucal de gestantes de uma comunidade carente do município de São Paulo: problemas percebidos e acesso a tratamento. Online Brazilian Journal of Nursing. 2008; 7(2).

14. FINKLER M, et al. Saúde bucal materno-infantil: Um estudo de representações sociais com gestantes. Texto Contexto Enferm., 2004; 13(3): 360-8.

15. FONSECA MRCC, et al. Prevalência do uso de medicamentos na gravidez: uma abordagem. Rev. Saúde Pública. 2002; 36(2).

16. GOEPEL E, et al. The need for cooperation between the gynecologist and dentist in pregnancy. A study of dental health education in pregnancy. Mar 1991.

17. LEAL NP e JANOTTI CB. Saúde bucal da gestante atendida no SUS: prática e representações de profissionais e pacientes. Feminina. 2009; 37(8): p. 413-421.

18. MAEDA FHL, et al. A visão das gestantes quanto às condutas odontológicas na cidade de Franca (SP). Rev Odontol., 2001; 3(2): 8-14.

19. MARTINS LO, et al. Assistência odontológica à gestante: percepção do cirurgião-dentista. Rev Pan-Amaz Saude. 2013; 4(4): 11-18.

20. MESQUITA BS, et al. Aspectos norteadores da atenção à saúde bucal de gestantes da estratégia saúde da família de João Pessoa, Brasil. Rev. Odontol. Bras. Central. 2013; 21(60). 
21. NASCIMENTO EP, et al. Gestantes frente ao tratamento odontológico. Rev. bras. odontol., 2012; 69(1): 125-30.

22. NOGUEIRA LT, et al. Retardo na procura do tratamento odontológico e percepção da saúde bucal em mulheres grávidas. Odontol. Clín. Cient., 2012.

23. ROSELL FL, et al. Impacto dos problemas de saúde bucal na qualidade de vida de gestantes. Pesq. Bras. Odontoped. Clin. Integr., 2013; 13(3): 287-93.

24. ROSSEL FL. Prevalência de fatores clínicos do risco de cárie em gestantes. Araraquara. Tese de Doutorado. Araraquara: Faculdade de Odontologia da Universidade Estadual Paulista "Júlio de Mesquita Filho"; 1998. $120 \mathrm{f}$.

25. ROSA PC, et al. Indicadores de saúde bucal de gestantes vinculadas ao programa de pré-natal em duas unidades básicas de saúde em Porto Alegre/RS. Arquivos em Odontologia. 2007; 43(01).

26. SANTOS CJGB. Atenção Odontológica à Gestante. Aracuaí - MG, 2010.

27. SCAVUZZI AIF, et al. Avaliação dos Conhecimentos e Práticas em Saúde Bucal de Gestantes Atendidas no Setor Público e Privado, em Feira de Santana, Bahia, Brasil. Pesq. Bras. Odontoped. Clin. Integr., João Pessoa, jan/abr. 2008.

28. SILVA ADM. Saúde Bucal de Gestantes: uma abordagem de gênero. In: Anais do 2ํㅡㄹ Congresso Mundial de Odontologia; 2001; 10-13.

29. SURESH L e RADFAR L. Medical management update. Pregnancy and lactation. Oral Surg. Oral Med. Oral Pathol. 2004.

30. TREVISAN CL, PINTO AAM. Fatores que Interferem no Acesso e na Adesão das Gestantes ao Tratamento Odontológico. Arch. Health Invest., 2013.

31. VIEIRA SM, et al. Percepção das puérperas sobre a assistência prestada pela equipe de saúde no prénatal. Texto contexto - enferm. 2011; 20 no.spe Florianópolis.

32. ZANROSSO CW, et al. N-Acetyltransferase 2 polymorphisms and susceptibility to infant leukemia with maternal exposure to dipyrone during pregnancy. Cancer Epidemiol Biomarkers, Prev. 2010. 